\title{
Preface : Invited Issue Editor, Professor Byung-Kyu Cho, and the Pediatric Epilepsy Surgery
}

\author{
Seung-Ki Kim, M.D., Ph.D., ${ }^{1,2}$ Kyu-Chang Wang, M.D., Ph.D. \\ Editor of the 'Pediatric Issue,", Journal of Korean Neurosurgical Society, Seoul, Korea \\ Chair, ${ }^{2}$ Publication Committee, The Korean Society for Pediatric Neurosurgery, Seoul, Korea
}

Since 2015, the Korean Society for Pediatric Neurosurgery (KSPN) has published an official journal, the Pediatric Issue as a supplement to the 'Journal of Korean Neurosurgical Society (JKNS), the official journal of the Korean Neurosurgical Society (KNS). The 'JKNS : Pediatric Issue' aims to share updated information on specific topics of pediatric neurosurgery with general neurosurgeons and those in other subspecialties, not just pediatric neurosurgeons. The review articles in the issue are subject to peer review to minimize any possible errors. Pediatric Issue is published in May each year particularly to honor Children's Day on May 5 and an annual meeting of KSPN held in May. One of the figures presented in the Pediatric Issue is selected to be on the cover of the May issue of the journal.

Pediatric Issue has made a significant contribution to the enhancement of the academic level of JKNS as well as pediatric neurosurgery by covering a wide range of topics from 2015 to 2018, as moyamoya disease (edited by Professor KyuChang Wang and Seung-Ki Kim), craniosynostosis (invited editor, Professor Joon-Ki Kang), the era of neuroendoscopy (invited editor, Professor Joong-Uhn Choi), and pediatric malignant brain cancers in the molecular era (invited editor, Professor James T. Rutka). The topic of 2019 'JKNS : Pediatric Is- sue,' 'Pediatric epilepsy surgery,' was organized by the invited Pediatric Issue editor, Professor Byung-Kyu Cho, one of the key figures who laid the foundation of pediatric neurosurgery in Korea (Fig. 1).

Professor Byung-Kyu Cho, born on December 11, 1944 graduated from Seoul National University College of Medicine in 1970. He was trained as a neurosurgical resident from 1971 to 1975 at Seoul National University Hospital and earned board certification in 1975. After completing his military duty

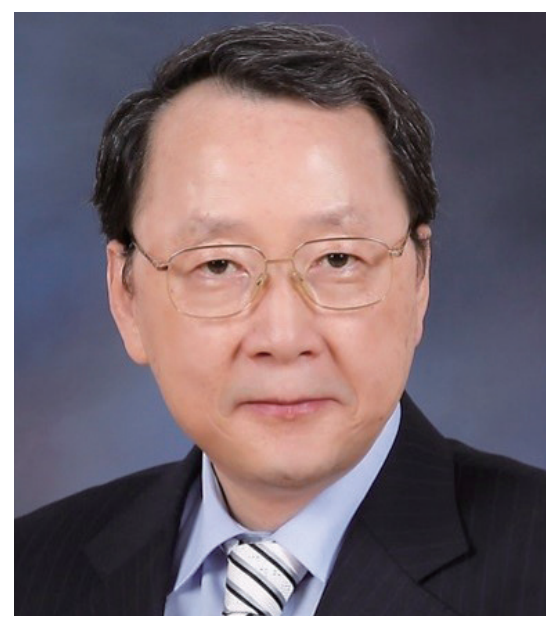

Fig. 1. Invited Issue Editor, Professor Byung-Kyu Cho.

- Received : January 14, 2019 •Accepted : January 26, 2019

-Address for reprints : Kyu-Chang Wang, M.D., Ph.D.

Division of Pediatric Neurosurgery, Seoul National University Children's Hospital, Seoul National University College of Medicine, 101 Daehak-ro, Jongno-gu, Seoul 03080, Korea Tel : +82-2-2072-3489, Fax : +82-2-2072-0274, E-mail : kcwang@snu.ac.kr

This is an Open Access article distributed under the terms of the Creative Commons Attribution Non-Commercial License (http://creativecommons.org/licenses/by-nc/4.0) which permits unrestricted non-commercial use, distribution, and reproduction in any medium, provided the original work is properly cited. 
(1975-1978), he became the Chairman of Department of Neurosurgery at Eulji General Hospital in Seoul and moved to the Department of Neurosurgery in Seoul National University Hospital where he worked until his retirement in 2010. Professor Cho studied as a research fellow at Children's Hospital of Philadelphia in the United States from 1983 to 1985 under the guidance of Professor Luis Schut. During the period, he was quite actively involved in the laboratory research of hydrocephalus, brain edema, evoked potentials and brain bioenergetics. After he returned to Korea, he served as the Chief of Division of Pediatric Neurosurgery at Seoul National University Children's Hospital (1985-1995) and the Chairman of Department of Neurosurgery at Seoul National University College of Medicine and Seoul National University Hospital (1996-2000). He played a key role in the establishment of the KSPN with Professors Joon-Ki Kang, Joong-Uhn Choi, and Hoon Kap Lee in 1987 and served as the President of KSPN from 1996 to 1998. Professor Cho displayed remarkable leadership while he was the President of the KNS (2000-2002). After he retired from Seoul National University, Professor Cho went to the Armed Forces Capital Hospital where he has been actively participating in patient care and brain surgery to this date.

Professor Cho is known to have inspired others with his 'gentleness,' 'concentration,' and 'perseverance,' especially his juniors who often express their heartfelt respect and gratitude for his faithfulness and continued support. His such characteristics are evident in the challenges he took on to perform the surgeries that had been considered to be too difficult to even dare to attempt. It was his sincere research on the abundant literature along with his own experience that enabled his success. Once, the surgery that he operated for 24 hours nonstop made a headline in a major newspaper.

Professor Cho made a great deal of academic contribution to the further improvement of his main interest fields; the pediatric brain tumors, moyamoya disease and epilepsy surgery. As one of the pioneers in the field of pediatric neuro-oncology in Korea, he introduced the concept of radical excision of brain tumors. While the extent of tumor removal is still one of the most critical factors in the prognosis of pediatric brain tumors, Professor Cho tried not to compromise the quality of the patient's life at the expense of the radical excision.
In 2010, he edited and published the monograph on moyamoya disease, 'Moyamoya Disease Update', with Professor Teiji Tominaga of Tohoku University in Japan. The 'Moyamoya Disease Update' contained the accumulated information on the moyamoya disease in terms of clinical practice and basic research. In 1995, he started epilepsy surgery on children with temporal lobe epilepsy. Ever since, he has treated many children with epileptic disorder and published cardinal papers about low grade tumor related seizure and focal cortical dysplasia. Professor Cho was invited to be the editor of Pediatric Issue of 2019 : pediatric epilepsy surgery, due to his such remarkable contribution to the field.

As recent advancement of neuroscience and the related modern technology has taken the field of diagnosis and treatment of epilepsy to a new level, the articles of this Pediatric Issue will undoubtedly provide the readers with current concepts and future directions of pediatric epilepsy surgery. Twelve articles were contributed by leading researchers in this field in Korea and the United States. Focusing on the new modern technology, Dr. Kennedy of the United States contributed an article on the clinical application of stereo-electroencephalography in seizure management. Dr. Lee of the United States provided a detailed analysis of the differences in epilepsy surgery between children and adults. Current and future perspectives were summarized at the end by Professor Cho.

We deeply appreciate all of the contributors, especially those from the United States and the professors of the multi-disciplinary team (Neuroscience, Pathology, and Pediatrics) for their active participation. Also, we would like to express our sincere gratitude to Professor Cho for his enthusiasm and dedication as the invited editor.

\section{CONFLICTS OF INTEREST}

No potential conflict of interest relevant to this article was reported.

\section{- Acknowledgements}

We deeply appreciate Ms. Bo-Young Lee for the English editing of this article. 